\title{
Novel methodology for localizing and studying the insect dorsal rim area morphology in 2D and 3D
}

\author{
Vun Wen Jie ${ }^{1}$, Arttu Miettinen²,3, Emily Baird ${ }^{1}$ \\ ${ }^{1}$ Dept. of Zoology, Stockholm University, Stockholm, Sweden \\ ${ }^{2}$ Department of Physics, University of Jyvaskyla, 40014, Jyvaskyla, Finland \\ ${ }^{3}$ Swiss Light Source, Paul Scherrer Institute, Villigen, Switzerland. \\ Correspondence: vun.wen.jie@zoologi.su.se
}

\section{Abstract}

Polarized light-based navigation in insects is facilitated by a specialised polarisation-sensitive part of the eye known as the dorsal rim area (DRA). Existing methods to study the anatomy of the DRA are in most cases destructive and very time consuming. Here, we present a novel method that allows for DRA localization using 3D volumetric images acquired through micro-computed tomography in combination with 2D photographs. We used the method on size polymorphic buff-tailed bumblebees, Bombus terrestris. We found that the size of the DRA can be easily acquired from photographs of the dorsal part of the eye. An allometric analysis of the size of the DRA in relation to body size in $B$. terrestris showed that the DRA region increases with body size but not at the same rate. Using our method, we localised the DRA in a 3D volume rendering of the eye using 2D photos localization of an individual's DRA onto the volumetric image stack of the head allowed for individual-level descriptions of the ommatidial structures (lens, crystalline cones, rhabdoms) to be performed on three different eye regions (DRA, non-DRA, proximate to DRA). The only feature distinctly unique to the DRA ommatidia was the smaller dimension of crystalline cones in comparison to other regions. Using our novel methodology we provide the first individual-level description of DRA ommatidial features and a comparison of how the DRA area varies with body size in bumblebees.

Keywords: Dorsal rim area, micro-computed tomography, virtual histology, ommatidia, allometry, bumblebee

\section{Introduction}

Navigation is pivotal for many animals to their day-to-day tasks of foraging, finding mates, avoiding competition or predation and returning home. While on such journeys, insects maintain their bearings using directional information acquired from the position of the sun (Dyer and Dickinson, 1994; Wehner, 1989; Wiltschko and Wiltschko, 1980), moon and stars (Dacke et al., 2004, 2003a; Nørgaard et al., 2008; Papi, 2001; Wehner, 1992), the spectral light distribution across the sky (Wehner, 1997) and landmarks (BischKnaden and Wehner, 2003; Bregy et al., 2008; Cartwright and Collett, 1983; Cheng et al., 2009; Knaden and Wehner, 2005; Seidl and Wehner, 2006; Steck et al., 2010, 2009; Wehner et al., 2006, 1996; Wehner, 2003, 1992). Insects can also obtain compass information using the pattern of polarization that is created in the sky (Frisch, 1967; Wehner, 1997; Wehner and Labhart, 2006) by the scattering of sunlight in the Earth's atmosphere (Brines and Gould, 1982; Stockhammer, 1959). While this pattern is invisible to us, it can be perceived by insects through a small dorsal subsection of their compound eyes called the dorsal rim area (DRA). The DRA ommatidia - the single visual units, or facets of a compound eye - are structurally different from those in non-DRA regions and contain orthogonally arranged microvilli that enable the perception of polarized light (Goldsmith and Wehner, 1977; Hardie, 1984; Israelachvili and Wilson, 1976).

The function of the DRA has been primarily studied by analysing its structural morphology, which has now been detailed in multiple insect orders: Blattodea (Labhart and Meyer, 1999), Coleoptera (Dacke et al., 2003b, 2002, 1999; Gokan, 1990, 1989; Labhart et al., 2009, 1992; Labhart and Meyer, 1999), Diptera (Hardie et al., 1989; Labhart and Meyer, 1999; Strausfeld and Wunderer, 1985; Wada, 1974a, 1974b; Wunderer and Smola, 1982), Hymenoptera (Aepli et al., 1985; Duelli and Wehner, 1973; Greiner et 
al., 2007; Helversen and Edrich, 1974; Herrling, 1976; Labhart, 1986; Meyer and Domanico, 1999; Meyer and Labhart, 1981; Narendra et al., 2016, 2013; Räber, 1979; Reid, 2010; Sommer, 1979; Zeil et al., 2014), Lepidoptera (Hämmerle and Kolb, 1996; Kolb, 1985; Labhart et al., 2009; Meinecke, 1981; Stalleicken et al., 2006), Odonata (Meyer and Labhart, 1993), Orthoptera (Egelhaaf and Dambach, 1983; Homberg and Paech, 2002; Labhart and Keller, 1992). Though these studies describe the DRA morphology of a particular species, they do not provide analyses of variation between individuals (see Labhart and Keller 1992 for an exception) or complete descriptions of each of the different ommatidial components within the DRA. This is due primarily to methodological limitations - the histological and electron microscopy methods typically used in these studies are destructive and are limited to non-isotropic analyses of structures in either the longitudinal or the transverse plane in each specimen. To better understand how DRA morphology varies both within and between species, and how this might ultimately relate to differences in functionality, methodologies that are capable of describing the ommatidial features within one specimen in both $2 \mathrm{D}$ and $3 \mathrm{D}$ are necessary.

To characterize the morphological features of the DRA, one must first localize and isolate it from the non-DRA regions of the compound eye. The localization of the DRA region can be done through the identification of orthogonally arranged microvilli (Egelhaaf and Dambach, 1983; Greiner et al., 2007; Hämmerle and Kolb, 1996; Meyer and Labhart, 1993; Narendra et al., 2013; Zeil et al., 2014). In species where it has been studied, the DRA is often visually distinct from other parts of the eye. For example, in locusts with light-coloured eyes, the DRA appears as a dark region and in insects with black eyes, such as bumblebees, the DRA is often also indicated by a lighter region (Aepli et al., 1985; Greiner et al., 2007; Labhart et al., 1992; Labhart and Keller, 1992; Meyer and Labhart, 1981). Here, we describe a new method that combines 2D photography with 3D data generated from micro-computed tomography (micro-CT) to localize and characterize the DRA in eyes where it is visually distinct. Our method begins by superimposing a 2D image of the distinct DRA region of a compound eye onto the respective 3D reconstructed head model. This not only enables the localization of the DRA region that is otherwise invisible in the 3D data but also facilitates detailed descriptions of DRA morphology in individual specimens. In addition, our approach enables an analysis of how the DRA ommatidia are distributed in 3D space, which can provide insights into how they sample the skylight polarization pattern, something may have important consequences for their function within and between insect species. We applied our methodology to the bumblebee Bombus terrestris, which was chosen for this study because they have dark eyes with a grey dorsal region (Zeil et al., 2014) and they exhibit size-polymorphism (Goulson, 2010). We use our data to explore if and how the size of the DRA region in $B$. terrestris varies with both body and eye size. We demonstrate that we can accurately localise the DRA region by mapping the 2D images of individual eyes onto 3D volumetric scans of the same eyes and using this to guide virtual segmentation. Our approach was validated by using transmission electron microscopy (TEM) to compare the features of the virtually segmented DRA structures with features in other areas of the compound eye. Using our method, we provide the first complete description of the 3D morphological structures in DRA ommatidia in an individual insect and the first analysis of how DRA surface area changes with eye size within a species.

\section{Material and Methods}

\subsection{Animal handling}

Bombus terrestris colonies were acquired from a commercial supplier (Koppert, Netherlands) and kept in incubators (Panasonic MIR Cooled Incubators, $123 \mathrm{~L}$ ) at $26^{\circ} \mathrm{C}$ in complete darkness. They were provided with $50 \%$ sugar water solution and fresh-frozen, organic pollen every two to three days (Naturprodukter, Raspowder Bipollen Ekologiskt bipollen).

Twenty workers of different sizes were randomly selected and assigned individual ID numbers. The selected individuals were first anesthetized with $\mathrm{CO}_{2}$ and then sacrificed using ethyl acetate. The thorax was photographed (Canon IXUS $220 \mathrm{HS}, 12.1$ ) and the inter-tegular distance (ITD), the distance between the two insertion points of the wings that can be used as a proxy for body size (Cane, 1987), was measured in pixels and converted to mm using FIJI (Version 1.8.0_172) (Schindelin et al., 2012). 


\subsection{Acquisition of 2D DRA images and surface area measurements}

A marked individual was placed in a 3D printed sample holder attached to the end of a micromanipulator (Figure $1 \mathrm{~A}$ ). The dorsal region of the left compound eye was viewed under a stereomicroscope with a camera attachment (Figure 1 B). After removing the pile (soft hair) around the ocelli using micro-scissors, a small grid paper for size calibration was attached onto the midsection of the head. The grid was attached using super glue leaving at least one ocellus uncovered. The DRA was illuminated using a light source that was positioned at a $45^{\circ}$ with respect to the DRA position (Figure $1 \mathrm{C} \& \mathrm{D}$ ).

The DRA and the grid paper were photographed (Canon EOS 70D) with a resolution of $1824 \times 2432$ pixels. The DRA surface area in 2D was calculated from the images using FIJI (Version 1.8.0_172) (Schindelin et al., 2012).

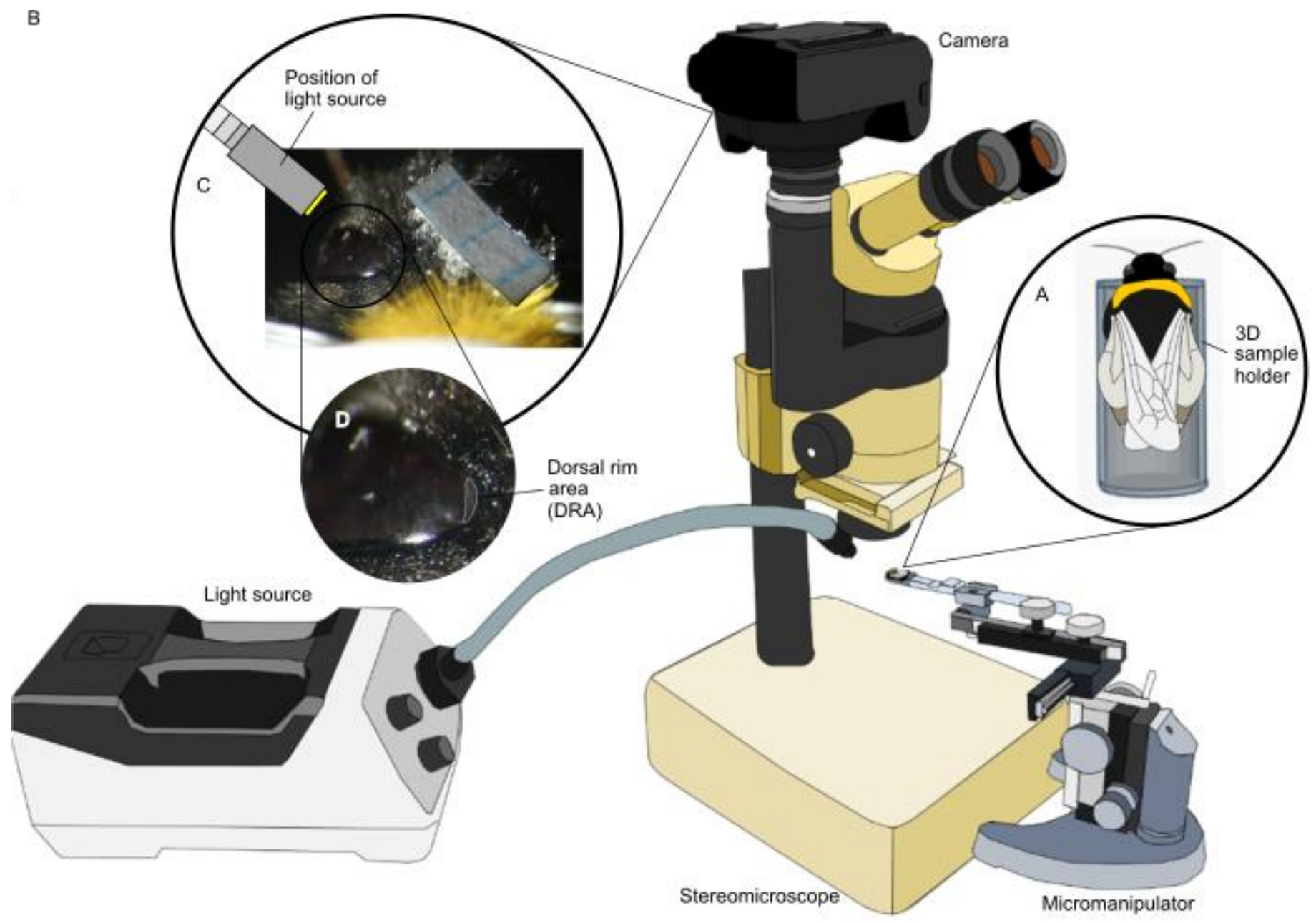

Figure 1: Setup and equipment for the dorsal rim area (DRA) image acquisition in 2D. (A) Bumblebee in sample holder, (B) Microscope set-up to acquire DRA photographs, (C) light set-up for DRA visualization, (D) enlarged view of the DRA.

\subsection{Staining and embedding}

The heads were dissected from the body and a microscalpel was used to remove the mouthparts. The heads were then immediately placed in $70 \%$ ethanol and $0.5 \%$ phosphotungstic acid (PTA) solution for 10 days (Smith et al., 2016). The heads underwent dehydration using a graded ethanol series and were cleared in acetone prior to transferring them to epoxy resin (Agar 100). Samples in the wet resin were transferred onto a perspex block and cured in an oven at $60^{\circ} \mathrm{C}$ for $48 \mathrm{~h}$. After curing, excess external resin was removed to expose the eyes and surrounding cuticle (Taylor et al., 2016). 


\subsection{Micro-CT}

Micro-CT scanning was performed at the TOMCAT beamline of the Swiss Light Source, Paul Scherrer Institute, Villigen (Switzerland) (beamtime number 20190641). Only 14 out of the selected 20 heads were scanned since the remaining 6 were damaged during staining and embedding. The intact heads were scanned using a monochromatic X-ray beam (20 keV), 2001 projection images of $2560 \times 2160$ pixels with a $60 \mathrm{~ms}$ exposure time were collected by a PCO.Edge 5.5 detector over 180 deg and a propagation distance of $100 \mathrm{~mm}$. The scans were done using a $4 x$ objective. The projection images were processed with the Paganin phase-retrieval method (Paganin et al., 2002) and reconstructed into 3D volumes using the gridrec algorithm (Marone and Stampanoni, 2012) with Parzen filter, resulting in 16-bit volume images that had an isotropic voxel size of $1.625 \mu \mathrm{m}$.

The regions of interest (ROIs) from the reconstructions were cropped using the program Drishti Paint (Limaye, 2012) and resaved as 16-bit TIFF files to reduce file size. Two ROIs were obtained from the reconstructions of each sample, and they consisted of (i) the left compound eyes (in the animal's perspective), and (ii) the dorsal head (the dorsal part of the left compound eye and the ocelli, which were used as landmarks to facilitate mapping the 2D images in later analysis, section 3.6).

\subsection{D Compound eye and surface area measurements}

3D models were generated from the cropped compound eyes in Amira (Version 2020.3.1, ThermoFisher Scientific) using a combination of segmenting tools that included thresholding, brush, fill, shrink and grow. The surface area of the compound eye was obtained by generating a surface from their segmented label (Taylor et al., 2019).

\subsection{Localizing the DRA on a 3D head model}

From the 14 dorsal head volumes, six with best-preserved eyes and high contrast micro-CT scans were chosen for DRA localization and anatomical ommatidial analysis. We chose two samples from each of the three chosen body size ranges based on the ITD, small $(2.965 \mathrm{~mm}-3.902 \mathrm{~mm})$, medium (3.902 mm $4.839 \mathrm{~mm})$ and large $(4.839 \mathrm{~mm}-5.776 \mathrm{~mm})$. 3D models of the eyes were generated in Amira using a combination of segmenting tools that included thresholding, brush, fill, shrink and grow. Because the grey DRA region is not visible in the 3D volumes, the localization of the DRA of an individual bumblebee requires both its $3 \mathrm{D}$ dorsal head and its $2 \mathrm{D}$ DRA image.

The DRA was localized by overlaying a 2D DRA image (Figure $1 \mathrm{C}$ ) onto the 3D dorsal head volume taken from the same specimen (Figure $2 \mathrm{~A}$ ). The ocelli were used as landmarks for precise mapping (Figure $2 \mathrm{~B}$ ). Mapping was performed in the project view of Amira using modules that included surface view and clipping plane. The DRA on the 3D model was segmented using the mapped image as a reference (Figure $2 \mathrm{C}$ ). The surface area of the DRA was obtained by generating a surface using the segmented DRA labels.

To determine the accuracy of our DRA localization method, we took transverse sections of the rhabdoms in regions predicted to be DRA and non-DRA on a separate individual prepared and visualised them using transmission electron microscopy (Romell et al., 2021). 


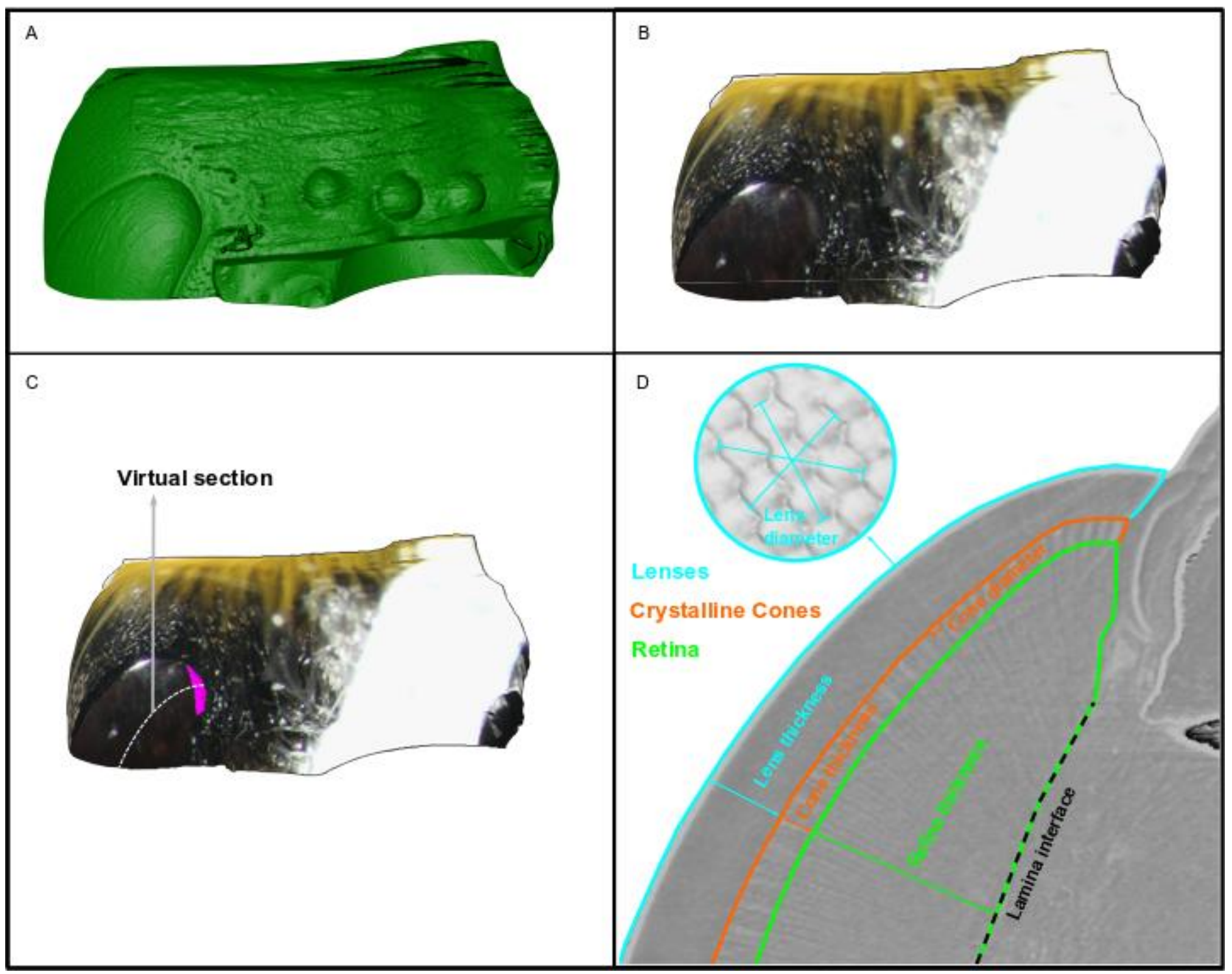

Figure 2: The process for localizing the DRA on a 3D head model of a bumblebee Bombus terrestris. (A) A 3D volume rendering of a bumblebee head. (B) A 2D photo is overlayed onto the $3 \mathrm{D}$ volume, $(\mathrm{C})$ The overlaid image is used to identify and segment the DRA (purple outline) in Amira. A grey dotted line indicates the region where virtual sectioning is performed. (D) Virtual slice showing ommatidial ommatidial structure measurements.

\subsection{DRA allometry and overall compound eye surface area}

Since the two methods used for determining the DRA surface area yielded very similar results (Figure 3), the surface area measurements of the DRA from the 2D photos were used for the allometric analysis of how DRA surface area varies with compound eye surface area $(n=20)$. The surface area of the compound eyes was obtained from the 3D compound eye models $(n=14)$. All variables were converted to linear measurements before logarithmic transformation. The relationships between the surface area of the DRA and compound eyes were plotted against ITD onto a log-log plot. 


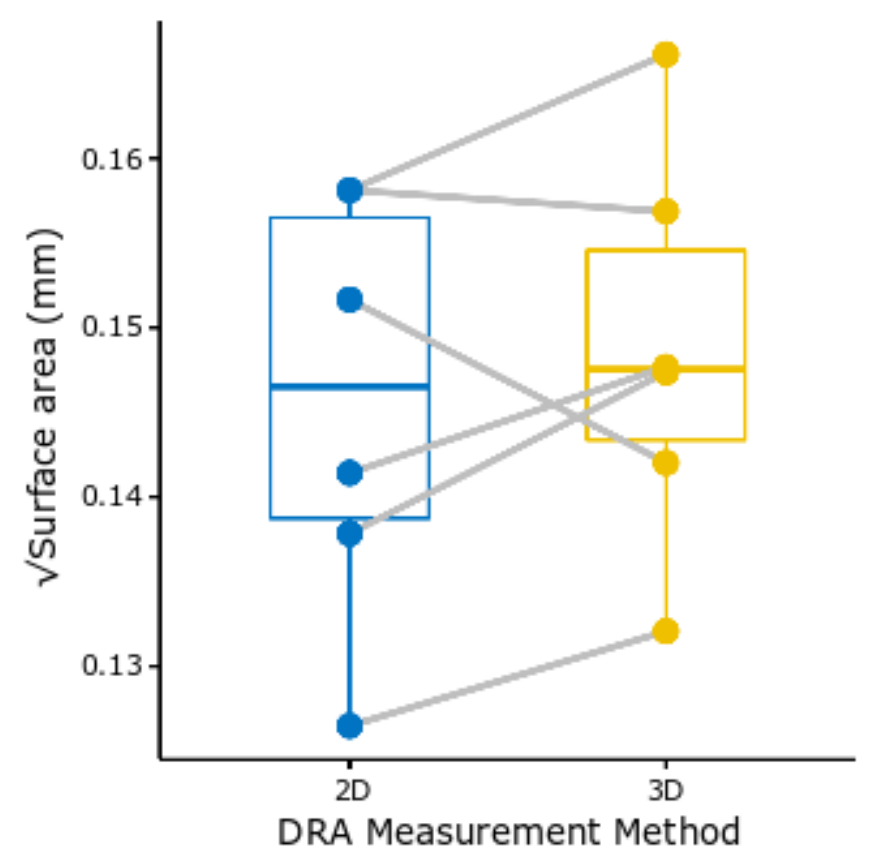

Figure 3: Boxplot comparing 2D and 3D DRA surface area measurements taken from the same individuals (grey lines) of Bombus terrestris. There was no significant difference between the values acquired using the 2D images or $3 \mathrm{D}$ volumes $(\mathrm{t}=-1.0421, \mathrm{p}=$ $0.3451, \mathrm{n}=6$, Wilcoxon signed-rank test).

\subsection{DRA ommatidial structure measurements}

The compound eyes from the six individuals that had their DRA localized and segmented were also used for volumetric analysis. The three chosen regions for ommatidial structure comparisons were within the DRA, proximate to the DRA (five ommatidial rows surrounding the DRA), and non-DRA ( $>20$ th ommatidial row vertically from the DRA). Seven longitudinal virtual histological slices consisting of the three regions were obtained per eye from the 3D scans using the clipping plane module in Amira. Only virtual slices where the rhabdoms were continuous were used for further analysis (Figure $2 \mathrm{D}$ ). Virtual histological slices were exported and analyzed in FIJI (Version 1.8.0_172) (Schindelin et al., 2012). The thickness of the lens, crystalline cones and rhabdoms as well as the width of the crystalline cones were measured. The lens width was measured externally on the model using 3D line tools (Figure $2 \mathrm{D}$ ). For each of the six eye samples, 20 ommatidial structure measurements were performed.

\section{Results and Discussion}

Our methodology allowed for the localization of the DRA on a homogeneous eye surface through the mapping of 2D images onto 3D volumetric data in individual compound eye samples and a comprehensive study of the gross internal and external morphology of the DRA structures. We discovered that external 2D images are sufficient for calculating the DRA surface area, confirming that this is a valid method for more accessible high-throughput analyses of DRAs within and between species.

\subsection{DRA localization on a homogeneous eye surface}

This is the first localization of the DRA through the mapping of 2D images onto 3D volumetric data generated from micro-CT scans. We used TEM to analyze the microvilli of transverse sections of DRA and 
non-DRA regions of the compound eye. The presence of orthogonally arranged microvilli in the region identified as the DRA suggests that our localization was accurate (Figure 4 A - B). In addition, since the structures are relatively flat, the comparative analysis between these and the $2 \mathrm{D}$ images showed that there are no significant differences between the method $(t=-1.0421, p=0.3451, n=6$, Wilcoxon signed-rank test) (Figure 3). This means that the 2D external images alone can provide an easy and high-throughput estimation of DRA surface area. Although the combination of photos and micro-CT data is not new (ljiri et al., 2018), we demonstrate here that it can also be used to make quantitative measurements. This methodology opens up new ways to study the DRA and other external features since it allows for individuallevel gross morphological descriptions of these structures.

\subsection{A comprehensive study of the internal and external morphology of DRA structures}

In contrast to previous methodology for characterising ommatidial structures in the DRA using TEM, our method allowed us to achieve gross morphological descriptions of most components of the DRA ommatidia within an individual insect due to its non-destructive nature. We found that the ommatidial structures are similar between areas within and proximate to DRA regions (Figure 4). The most distinct difference that we observed between the DRA and other eye regions was the thickness of crystalline cones; those in the DRA are both thinner (DRA vs proximate to DRA: $\mathrm{H}_{2}=10.211, \mathrm{p}=0.0223$; DRA vs non-DRA: $\mathrm{H}_{2}=10.211, \mathrm{p}=$ $0.0065, \mathrm{n}=6$, Pairwise Wilcoxon Rank Sum) and smaller in diameter (DRA vs proximate to DRA, $\mathrm{H}_{2}=$ 13.93, $p=0.0087$; DRA vs non-DRA: $\mathrm{H}_{2}=13.93, \mathrm{p}=0.0065, \mathrm{n}=6$, Pairwise Wilcoxon Rank Sum) than those in other regions. This is unlike what has been observed in other insects, such as the Canarian tiny cricket (Egelhaaf and Dambach, 1983) and the desert locust (Homberg and Paech, 2002). The question arises to as why some insects have distinct differences between the DRA and regions proximate to it while others do not? The comprehensiveness of this methodology allowed us to pick up minute differences between regions of interest and showed that structures within the DRA and proximate to the DRA can be very similar, at least in Bombus terrestris. 
A

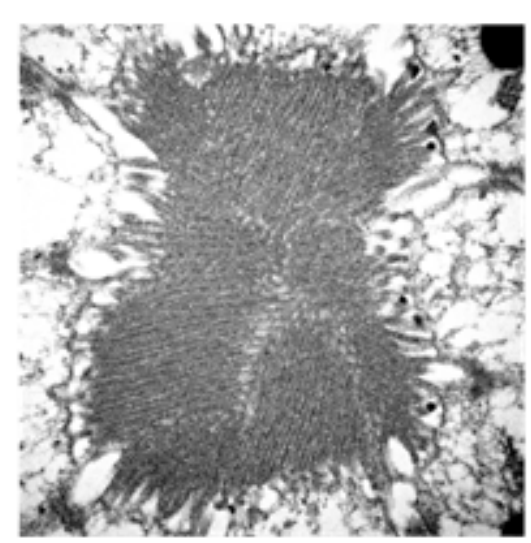

B

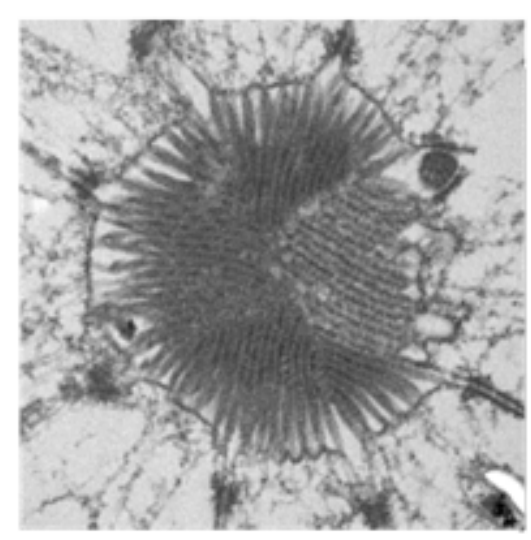

C

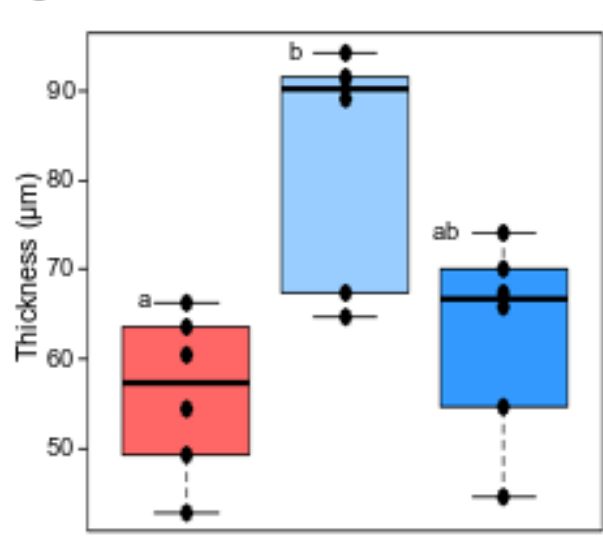

D

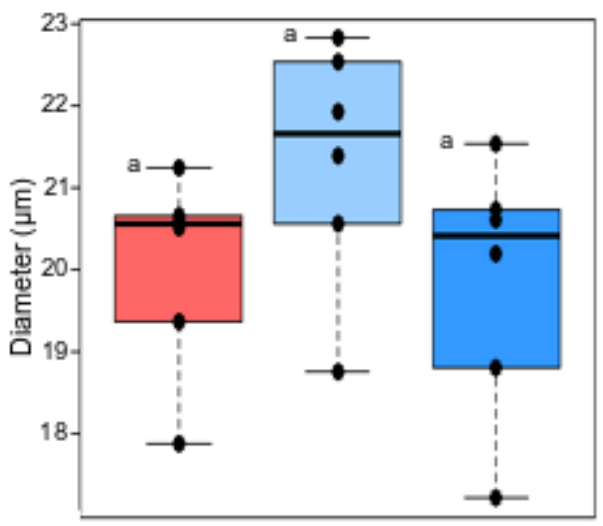

$\mathrm{E}$

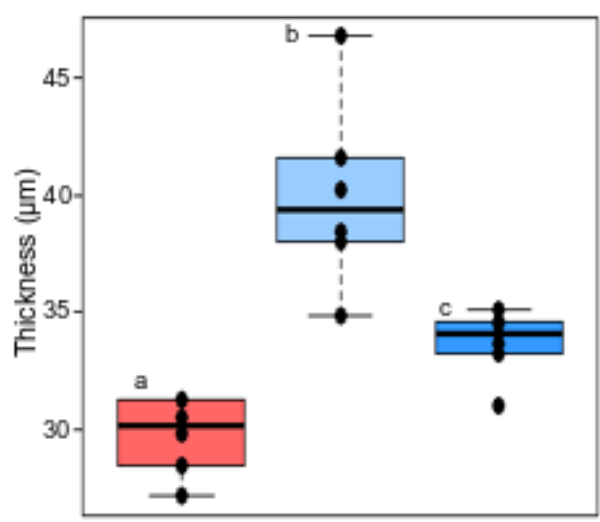

$\mathrm{H}$

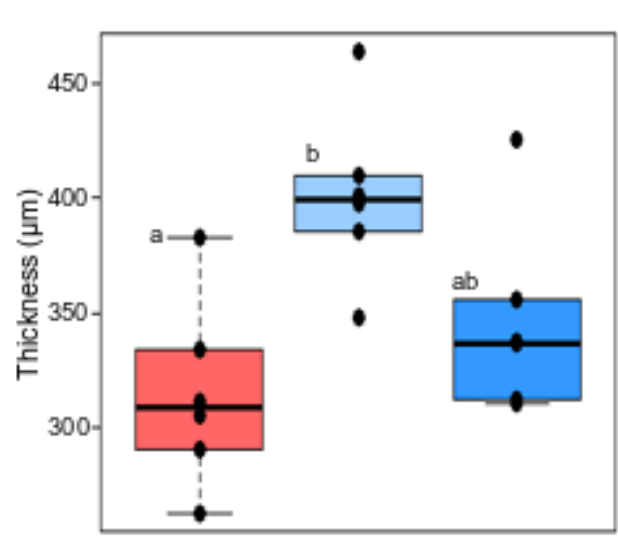

Ommatidia
$\mathrm{F}$

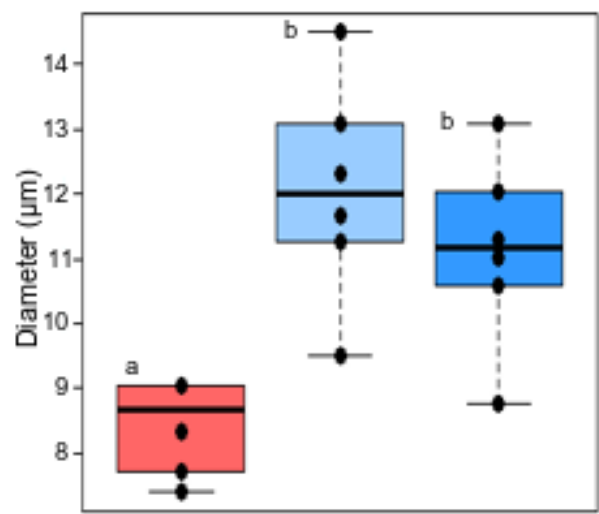

G

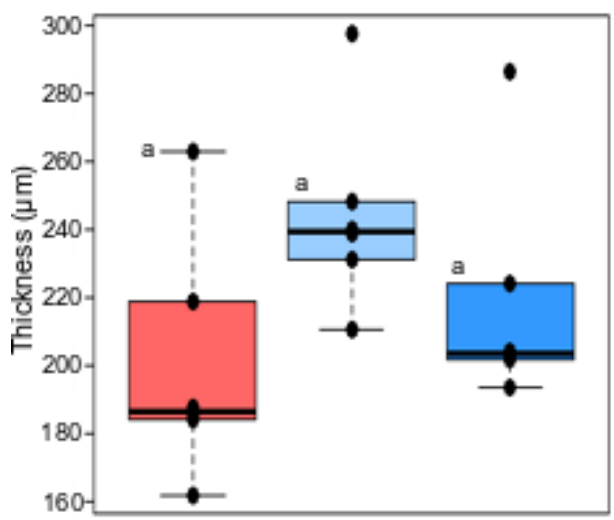

\section{Rhabdom}

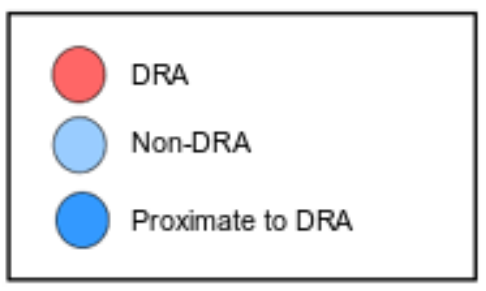

Figure 4: Transverse section of (A) DRA, and (B non-DRA microvilli $(n=1)$. Ommatidial structure comparison between DRA, proximate to the DRA and non-DRA regions. Thickness of lens (C), crystalline cones $(E)$, rhabdom $(G)$, and ommatidia $(H)$. Diameter of lens $(D)$, and crystalline cones $(F)(n=6)$. Letters represent the outcome of pairwise Wilcoxon Rank Sum tests, matching letters indicate no significant difference at $p=0.05$ and non-matching letters indicate significance below this value. 


\subsection{Method implementation on Bombus terrestris to study how the DRA varies with body size}

This study provided an easy and high-throughput methodology for studying the DRA surface area in insects that allowed us to perform the first allometric study of how surface area of the DRA scales with body size in bumblebees. Our results revealed that the DRA, as a small component of the compound eye, correlates positively with body size $\left(t_{12}=2.682, p=0.0152, n=20\right.$, regression analysis) (Figure 5$)$. The compound eye also correlates positively with body size $\left(\mathrm{t}_{12}=7.137, \mathrm{p}<0.001, \mathrm{n}=20\right.$, regression analysis). This result is consistent with the findings of Taylor et al. (Taylor et al., 2019) and suggests that the variation of eye size with body size likely affects visual capabilities such as resolution and sensitivity in $B$. terrestris. This, in turn, is likely to affect visually guided behavior, such as the timing of activity (Spaethe and Chittka, 2003; Spaethe and Weidenmüller, 2002). Do differences in DRA size between individuals observed here also relate to functional differences, for example in navigational capabilities (by affecting the sensitivity to polarised light in the DRA)? Our method allows questions like this to be answered by facilitating the quantification of the effect of body size on the size of the DRA and could ultimately inspire more questions about DRA functionality that can be tested behaviourally.

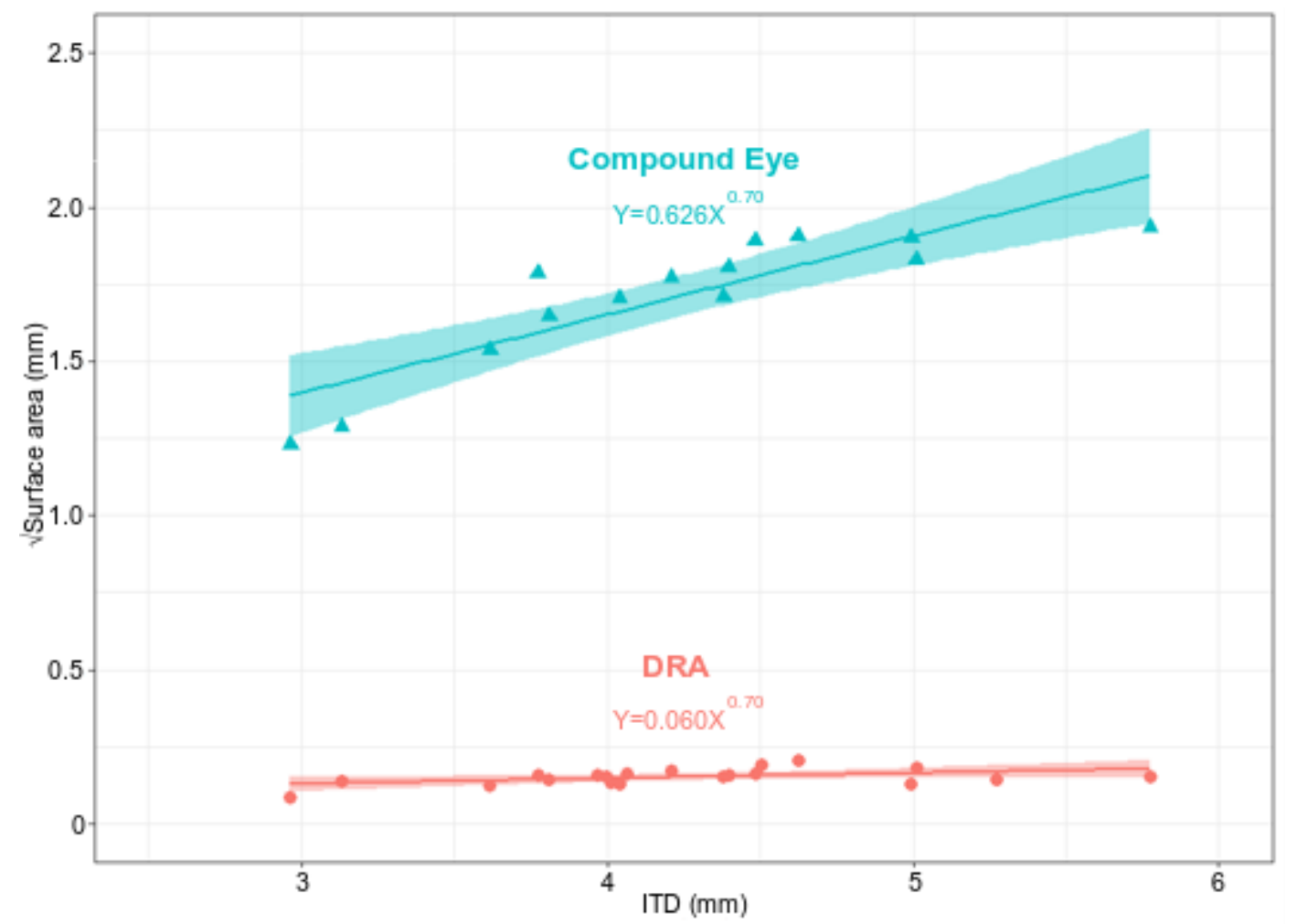

Figure 5: Allometric relationship between the square root of eye surface area $(\mathrm{mm})$ with ITD $(\mathrm{mm})$. Triangle $=3 \mathrm{D}$ compound eye measurements $\left(\mathrm{t}_{12}=2.682\right.$, $\mathrm{p}=0.0152, \mathrm{n}=14$, regression analysis $)$, Circle $=2 \mathrm{D}$ DRA measurements $\left(\mathrm{t}_{12}=\right.$ 7.137, $\mathrm{p}<0.001, \mathrm{n}=20$, regression analysis).

\subsection{Limitations of the method}

While our method provides new insights into the morphological structures of the DRA, both externally and internally within one individual, it has some limitations that are important to highlight. Although micro-CT based 3D volume renderings of compound eyes allow for a more comprehensive gross morphological description of the DRA structure, it does not have the resolution necessary for resolving the orientation of individual microvilli. This limitation should be addressed in the future as microvilli orientations within the DRA determine how the polarisation pattern of the sky is sampled. However, as the samples prepared for micro-CT are embedded in resin and stained with heavy metal they are also appropriate for performing TEM (Handschuh et al., 2013). This correlative approach would allow for the mapping of the microvilli 
arrangement at the individual-level that, to our knowledge, has never been achieved in 3D. A further limitation of our method is that the 3D methodology takes a long time to learn, requires advanced amounts of computing power (gigabytes of RAM), storage space (data range in gigabytes per dataset), and can be expensive. The 2D methodology, though it is considered high throughput only works if the DRA structures are relatively flat, which is the case in Bombus terrestris. This is not the case in butterflies as their DRAs are curved (ranging from the edge of the eye next to the antennae to the dorsal edge of the eye) (Stalleicken et al., 2006). To acquire the surface measurements of these, the 3D localization method is necessary. Finally, this method is limited to analysing the DRAs of insects with dark eyes and whose DRA is marked by a grey region.

\section{Conclusion}

To understand insect navigational behaviour, it is necessary to study all sensory structures involved, including the DRA that is linked to polarization vision-based navigation. Though this structure is widespread among insects, it has proven to be problematic to study, due to lack of complete and non-destructive individual-level methods. Our novel methodology presented in this study allows us to produce more insightful observations of DRA morphology and, in turn, raise questions about polarization vision-based navigation in insects.

\section{Acknowledgement}

We would like to thank Inga Tuminaite for assistance throughout the project and for critically reading the manuscript drafts. We would also like to acknowledge the Paul Scherrer Institut, Villigen, Switzerland for provision of synchrotron radiation beamtime at the TOMCAT beamline X02DA of the SLS. Pierre Tichit for his technical guidance in Amira. Julia Meneghello for helping in segmenting the heads and eye surface area of the bumblebees.

\section{Funding}

This work was supported by a grant from the Swedish Research Council (2018-06238).

\section{References}

1. Aepli, F., Labhart, T., Meyer, E.P., 1985. Structural specializations of the cornea and retina at the dorsal rim of the compound eye in hymenopteran insects. Cell Tissue Res. 239, 19-24.

2. Bisch-Knaden, S., Wehner, R., 2003. Local vectors in desert ants: Context-dependent landmark learning during outbound and homebound runs. J. Comp. Physiol. A Neuroethol. Sensory, Neural, Behav. Physiol. 189, 181-187.

3. Bregy, P., Sommer, S., Wehner, R., 2008. Nest-mark orientation versus vector navigation in desert ants. J. Exp. Biol. 211, 1868-1873.

4. Brines, M.L., Gould, J.L., 1982. Skylight Polarization patterns and Animal Orientation. J. Exp. Biol. 96, 69-91.

5. Cane, J.H., 1987. Estimation of Bee Size Using Intertegular Span ( Apoidea ). J. Kansas Entomol. Soc. 60, 145-147.

6. Cartwright, B.A., Collett, T.S., 1983. Landmark learning in bees - Experiments and models. J. Comp. Physiol. A 151, 521-543.

7. Cheng, K., Narendra, A., Sommer, S., Wehner, R., 2009. Traveling in clutter: Navigation in the Central Australian desert ant Melophorus bagoti. Behav. Processes 80, 261-268.

8. Dacke, M., Byrne, M.J., Scholtz, C.H., Warrant, E.J., 2004. Lunar orientation in a beetle. Proc. R. Soc. B Biol. Sci. 271, 361-365.

9. Dacke, M., Nilsson, D.E., Scholtz, C.H., Byrne, M., Warrant, E.J., 2003a. Insect orientation to polarized moonlight. Nature 424, 33.

10. Dacke, M., Nordström, P., Scholtz, C., 1999. Two sets of compound eyes in dungbeetles Pachysoma: a specialization for skylight navigation. In: Proceedings of the Göttingen Neurobiology Conference. p. 414.

11. Dacke, M., Nordström, P., Scholtz, C.H., 2003b. Twilight orientation to polarised light in the crepuscular dung beetle Scarabaeus zambesianus. J. Exp. Biol. 206, 1535-1543. 
bioRxiv preprint doi: https://doi.org/10.1101/2022.02.07.479135; this version posted February 9,2022 . The copyright holder for this preprint (which was not certified by peer review) is the author/funder. All rights reserved. No reuse allowed without permission.

12. Dacke, M., Nordström, P., Scholtz, C.H., Warrant, E.J., 2002. A specialized dorsal rim area for polarized light detection in the compound eye of the scarab beetle Pachysoma striatum. J. Comp. Physiol. A Neuroethol. Sensory, Neural, Behav. Physiol. 188, 211-216.

13. Duelli, P., Wehner, R., 1973. The spectral sensitivity of polarized light orientation in Cataglyphis bicolor (Formicidae, Hymenoptera). J. Comp. Physiol. 86, 37-53.

14. Dyer, F.C., Dickinson, J.A., 1994. Development of sun compensation by honeybees: How partially experienced bees estimate the sun's course. Proc. Natl. Acad. Sci. U. S. A. 91, 4471-4474.

15. Egelhaaf, A., Dambach, M., 1983. Giant rhabdomes in a specialized region of the compound eye of a cricket: Cycloptiloides canariensis (Insecta, Gryllidae). Zoomorphology 102, 65-77.

16. Frisch, K. Von, 1967. The Dance Language and Language and Orientation of Bees.

17. Gokan, N., 1989. The Compound Eye of the Dung Beetle Geotrupes auratus (Coleoptera: Scarabaeidae). Appl. Entomol. Zool. 24, 133-146.

18. Gokan, N., 1990. The compound eye of the dung, Onthophagus posticus (Coleoptera: Scarabaeidae). New Zeal. Entomol. 13, 7-15.

19. Goldsmith, T.H., Wehner, R., 1977. Restrictions on rotational and translational diffusion of pigment in the membranes of a rhabdomeric photoreceptor. J. Gen. Physiol. 70, 453-490.

20. Goulson, D., 2010. Social Organization and Conflict. In: Bumblebees, Behvaiour, Ecology, and Conservation. Oxford University Press, pp. 21-43.

21. Greiner, B., Cronin, T.W., Ribi, W.A., Wcislo, W.T., Warrant, E.J., 2007. Anatomical and physiological evidence for polarisation vision in the nocturnal bee Megalopta genalis. J. Comp. Physiol. A Neuroethol. Sensory, Neural, Behav. Physiol. 193, 591-600.

22. Hämmerle, B., Kolb, G., 1996. retinal ultrastructure of the dorsal eye region of Pararge Aegeria (Linne) (Lepidoptera: Satyridae). Int. J. Insect Morphol. Embryol. 25, 305-315.

23. Handschuh, S., Baeumler, N., Schwaha, T., Ruthensteiner, B., 2013. A correlative approach for combining microCT, light and transmission electron microscopy in a single 3D scenario. Front. Zool. $10,1-16$.

24. Hardie, R., Vogt, K., Ruwlph, A., 1989. The compound eyes of the tsetse fly (Glossina Morsitans Morsitans and Glossina Palpalis Palpalis). J. Insect Physiol. 35, 423-431.

25. Hardie, R.C., 1984. Properties of photoreceptors R7 and R8 in dorsal marginal ommatidia in the compound eyes of Musca and Calliphora. J. Comp. Physiol. A 154, 157-165.

26. Helversen, O. von, Edrich, W., 1974. The spectral sensitivity of ultraviolet light orientation in the honey bee. J. Comp. Physiol.

27. Herrling, P.L., 1976. Regional distribution of three ultrastructural retinula types in the retina of Cataglyphis bicolor Fabr. (Formicidae, Hymenoptera). Cell Tissue Res. 169, 247-266.

28. Homberg, U., Paech, A., 2002. Ultrastructure and orientation of ommatidia in the dorsal rim area of the locust compound eye. Arthropod Struct. Dev. 30, 271-280.

29. Ijiri, T., Todo, H., Hirabayashi, A., Kohiyama, K., Dobashi, Y., 2018. Digitization of natural objects with micro CT and photographs. PLoS One 13, 1-18.

30. Israelachvili, J., Wilson, M., 1976. Absorption Characteristics of Oriented Photopigments in Microvilli. Biol. Cybern. 21, 9-15.

31. Knaden, M., Wehner, R., 2005. Nest mark orientation in desert ants Cataglyphis: What does it do to the path integrator? Anim. Behav. 70, 1349-1354.

32. Kolb, G., 1985. Ultrastructure and adaptation in the retina of Aglais urticae (Lepidoptera). Zoomorphology 105, 90-98.

33. Labhart, T., 1986. The electrophysiology of photoreceptors in different eye regions of the desert ant, Cataglyphis bicolor. J. Comp. Physiol. A 1-7.

34. Labhart, T., Baumann, F., Bernard, G.D., 2009. Specialized ommatidia of the polarization-sensitive dorsal rim area in the eye of monarch butterflies have non-functional reflecting tapeta. Cell Tissue Res. 338, 391-400.

35. Labhart, T., Keller, K., 1992. Fine Structure and Growth of the Polarization- Sensitive Dorsal Rim Area in the Compound Eye of Larval Crickets. Naturwissenschaften 79, 79, 527-529.

36. Labhart, T., Meyer, E.P., 1999. Detectors for polarized skylight in insects: A survey of ommatidial specializations in the dorsal rim area of the compound eye. Microsc. Res. Tech. 47, 368-379.

37. Labhart, T., Meyer, E.P., Schenker, L., 1992. Specialized ommatidia for polarization vision in the compound eye of cockchafers, Melolontha melolontha (Coleoptera, Scarabaeidae). Cell Tissue Res. 268, 419-429.

38. Limaye, A., 2012. Drishti: a volume exploration and presentation tool. Dev. X-Ray Tomogr. VIII 8506, 85060X.

39. Marone, F., Stampanoni, M., 2012. Regridding reconstruction algorithm for real-time tomographic imaging. J. Synchrotron Radiat. 19, 1029-1037. 
bioRxiv preprint doi: https://doi.org/10.1101/2022.02.07.479135; this version posted February 9,2022 . The copyright holder for this preprint (which was not certified by peer review) is the author/funder. All rights reserved. No reuse allowed without permission.

40. Meinecke, C.C., 1981. The Fine Structure of the Compound Eye of the African Armyworm Moth, Spodoptera exempta Walk. (Lepidoptera, Noctuidae). Cell Tissue Res. 12, 333-347.

41. Meyer, E.P., Domanico, V., 1999. Microvillar orientation in the photoreceptors of the ant Cataglyphis bicolor. Cell Tissue Res. 295, 355-361.

42. Meyer, E.P., Labhart, T., 1981. Pore canals in the cornea of a functionally specialized area of the honey bee's compound eye. Cell Tissue Res. 216, 491-501.

43. Meyer, E.P., Labhart, T., 1993. Morphological specializations of dorsal rim ommatidia in the compound eye of dragonflies and damselfies (Odonata). Cell Tissue Res. 272, 17-22.

44. Narendra, A., Alkaladi, A., Raderschall, C.A., Robson, S.K.A., Ribi, W.A., 2013. Compound Eye Adaptations for Diurnal and Nocturnal Lifestyle in the Intertidal Ant, Polyrhachis sokolova. PLoS One 8, 4-9.

45. Narendra, A., Ramirez-Esquivel, F., Ribi, W.A., 2016. Compound eye and ocellar structure for walking and flying modes of locomotion in the Australian ant, Camponotus consobrinus. Sci. Rep. 6 , $1-10$.

46. Nørgaard, T., Nilsson, D.E., Henschel, J.R., Garm, A., Wehner, R., 2008. Vision in the nocturnal wandering spider Leucorchestris arenicola (Araneae: Sparassidae). J. Exp. Biol. 211, 816-823.

47. Paganin, D., Mayo, S.C., Gureyev, T.E., Miller, P.R., Wilkins, S.W., 2002. Simultaneous phase and amplitude extraction from a single defocused image of a homogeneous object. J. Microsc. 206, 3340.

48. Papi, F., 2001. Animal navigation at the end of the century: A retrospect and a look forward. Ital. J. Zool. 68, 171-180.

49. Räber, F.W., 1979. Retinatopographie und Sehfeldtopologie des Komplexauges von Cataglyphis bicolor (Formicidae, Hymenoptera) und einiger verwandter Formiciden-Arten. Uitgever niet vastgesteld.

50. Reid, S.F., 2010. Life in the dark: Vision and navigation in a nocturnal bull ant. The Australian National University.

51. Romell, J., Jie, V.W., Miettinen, A., Baird, E., Hertz, H.M., 2021. Laboratory phase-contrast nanotomography of unstained Bombus terrestris compound eyes. J. Microsc. 283, 29-40.

52. Schindelin, J., Arganda-Carreras, I., Frise, E., Kaynig, V., Longair, M., Pietzsch, T., Preibisch, S., Rueden, C., Saalfeld, S., Schmid, B., Tinevez, J.Y., White, D.J., Hartenstein, V., Eliceiri, K., Tomancak, P., Cardona, A., 2012. Fiji: An open-source platform for biological-image analysis. Nat. Methods 9, 676-682.

53. Seidl, T., Wehner, R., 2006. Visual and tactile learning of ground structures in desert ants. J. Exp. Biol. 209, 3336-3344.

54. Smith, D.B., Bernhardt, G., Raine, N.E., Abel, R.L., Sykes, D., Ahmed, F., Pedroso, I., Gill, R.J., 2016. Exploring miniature insect brains using micro-CT scanning techniques. Sci. Rep. 6.

55. Sommer, E.W., 1979. Untersuchungen zur topographischen Anatomie der Retina und zur Sehfeldtopologie im Auge der Honigbiene, Apis mellifera (Hymenoptera). Universität Zürich.

56. Spaethe, J., Chittka, L., 2003. Interindividual variation of eye optics and single object resolution in bumblebees. J. Exp. Biol. 206, 3447-3453.

57. Spaethe, J., Weidenmüller, A., 2002. Size variation and foraging rate in bumblebees (Bombus terrestris). Insectes Soc. 49, 142-146.

58. Stalleicken, J., Labhart, T., Mouritsen, H., 2006. Physiological characterization of the compound eye in monarch butterflies with focus on the dorsal rim area. J. Comp. Physiol. A Neuroethol. Sensory, Neural, Behav. Physiol. 192, 321-331.

59. Steck, K., Knaden, M., Hansson, B.S., 2010. Do desert ants smell the scenery in stereo? Anim. Behav. 79, 939-945.

60. Steck, K., Wittlinger, M., Wolf, H., 2009. Estimation of homing distance in desert ants, Cataglyphis fortis, remains unaffected by disturbance of walking behaviour. J. Exp. Biol. 212, 2893-2901.

61. Stockhammer, K., 1959. Die Orientierung nach der Schwingungsrichtung linear polarisierten Lichtes und ihre sinnesphysiologischen Grundlagen. In: Ergebnisse Der Biologie. Springer, pp. 23-56.

62. Strausfeld, N.J., Wunderer, H., 1985. Optic lobe projections of marginal ommatidia in Calliphora erythrocephala specialized for detecting polarized light. Cell Tissue Res. 242, 163-178.

63. Taylor, G.J., Ribi, W., Bech, M., Bodey, A.J., Rau, C., Steuwer, A., Warrant, E.J., Baird, E., 2016. The dual function of orchid bee ocelli as revealed by X-ray microtomography. Curr. Biol. 26, 13191324.

64. Taylor, G.J., Tichit, P., Schmidt, M.D., Bodey, A.J., Rau, C., Baird, E., 2019. Bumblebee visual allometry results in locally improved resolution and globally improved sensitivity. Elife 8, 1-32.

65. Wada, S., 1974a. Spezielle randzonale ommatidien der fliegen (diptera: brachycera): architektur und verteilung in den komplexauaen. Zeitschrift für Morphol. der Tiere 77, 87-125. 
66. Wada, S., 1974b. Spezielle randzonale ommatidien von Calliphora Erythrocephala meig (Diptera: Calliphoridae): Architektur der zentralen rhabdomeren-kolumne und topographie im komplexauge. Int. J. Insect Morphol. Embryol. 3, 397-424.

67. Wehner, R., 1989. The Hymenopteran Skylight Compass: Matched Filtering and Parallel Coding. J. Exp. Biol. 146, 63-85.

68. Wehner, R., 1992. Arthropods. In: Papi, F. (Ed.), Animal Homing. Springer, pp. 45-144.

69. Wehner, R., 1997. The ant's celestial compass system: spectral and polarization channelss. In: Lehrer, M. (Ed.), Orientation and Communication in Arhtropods. Springer, pp. 145-185.

70. Wehner, R., 2003. Desert ant navigation: How miniature brains solve complex tasks. J. Comp. Physiol. A Neuroethol. Sensory, Neural, Behav. Physiol. 189, 579-588.

71. Wehner, R., Boyer, M., Loertscher, F., Sommer, S., Menzi, U., 2006. Ant navigation: One-way routes rather than maps. Curr. Biol. 16, 75-79.

72. Wehner, R., Labhart, T., 2006. Polarisation vision. In: Warrant, E., Nilsson, D.-E. (Eds.), Invertebrate Vision. Cambridge University Press, pp. 291-348.

73. Wehner, R., Michel, B., Antonsen, P., 1996. Visual navigation in insects: Coupling of egocentric and geocentric information. J. Exp. Biol. 199, 129-140.

74. Wiltschko, R., Wiltschko, W., 1980. The process of learning sun compass orientation in young homing pigeons. Naturwissenschaften $67,512-514$.

75. Wunderer, H., Smola, U., 1982. Fine structure of ommatidia at the dorsal eye margin of Calliphora erythrocephala meigen (Diptera: Calliphoridae): An eye region specialised for the detection of polarized light. Int. J. Insect Morphol. Embryol. 11, 25-38.

76. Zeil, J., Ribi, W., Nerandra, A., 2014. Polarisation vision in Ants, Bees and wasps. In: Horvath, G., Marshall, J., Collin, S. (Eds.), Polarized Light and Polarization Vision in Animal Sciences. Springer, pp. 41-60. 